\title{
Elevated Blood Urea Nitrogen/Creatinine Ratio Is Associated with Venous Thromboembolism in Patients with Acute Ischemic Stroke
}

\author{
Hoon Kim, M.D., Ph.D., ${ }^{1}$ Kiwon Lee, M.D., FACP, FAHA, FCCM, ${ }^{2}$ Huimahn A. Choi, M.D., ${ }^{2}$ Sophie Samuel, Pharm.D., BCPS, ${ }^{3}$ \\ Jung Hyn Park, M.D., ${ }^{4}$ Kwang Wook Jo, M.D., Ph.D. \\ Department of Neurosurgery, Bucheon St. Mary's Hospital, College of Medicine, The Catholic University of Korea, Bucheon, Korea \\ Department of Neurosurgery and Neurology, ${ }^{2}$ The University of Texas Medical School, Houston, TX, USA \\ Department of Pharmacy, Memorial Hermann - Texas Medical Center, Houston, TX, USA \\ Department of Neurosurgery, ${ }^{4}$ Dongtan Sacred Heart Hospital, Hallym University Medical Center, Hwaseong, Korea
}

Objective : Although venous thromboembolism (VTE) is frequently related to dehydration, the impact of dehydration on VTE in acute ischemic stroke (AIS) is not clear. This study investigated whether dehydration, as measured by blood urea nitrogen (BUN)/ creatinine (Cr) ratio, influences the occurrence of VTE in patients with AIS.

Methods : This is a retrospective study of patients with AIS between January 2012 and December 2013. Patients with newly diagnosed AIS who experienced prolonged hospitalization for at least 4 weeks were included in this study.

Results : Of 182 patients included in this study, 17 (9.3\%) suffered VTE during the follow-up period; in two cases, VTE was accompanied by deep vein thrombosis and pulmonary embolism. Patients with VTE were more frequently female and had higher National Institutes of Health Stroke Scale (NIHSS) score, more lower limb weakness, and elevated blood urea nitrogen BUN/Cr ratio on admission. In a multivariate analysis, BUN/Cr ratio $>15$ (odds ratio [OR] 8.75) and severe lower limb weakness (OR 4.38) were independent risk factors for VTE.

Conclusion : Dehydration on admission in cases of AIS might be a significant independent risk factor for VTE.

Key Words : Blood urea nitrogen · Creatinine · Venous thrombosis · Dehydration · Cerebral infarction.

\section{INTRODUCTION}

Venous thromboembolism (VTE) is a serious and potentially fatal complication of acute ischemic stroke (AIS) ${ }^{8,30,33)}$. The incidence of deep vein thrombosis (DVT) during the first two weeks after stroke ranges from $27 \%$ to $75 \%$ in untreated patients ${ }^{14)}$. Pulmonary embolism (PE) is the most common cause of death between the first two and four weeks after $\mathrm{AIS}^{17}$. Because DVT is often asymptomatic in AIS patients, it is important to identify risk factors capable of predicting the occurrence of DVT. In previous studies, risk factors for DVT included comorbidities and neurological deficits such as older

- Received : October 29, 2016 • Revised : January 25, 2017 •Accepted : March 14, 2017

- Address for reprints : Kwang Wook Jo, M.D., Ph.D.

Department of Neurosurgery, Bucheon St. Mary's Hospital, College of Medicine, The Catholic University of Korea, 327 Sosa-ro, Wonmi-gu, Bucheon 14647, Korea Tel : +82-32-340-2259, Fax : +82-32-340-7391, E-mail : jkw94@naver.com

This is an Open Access article distributed under the terms of the Creative Commons Attribution Non-Commercial License (http://creativecommons.org/licenses/by-nc/4.0) which permits unrestricted non-commercial use, distribution, and reproduction in any medium, provided the original work is properly cited. 
age, atrial fibrillation, and a higher degree of limb paresis ${ }^{18,22)}$. Additionally, prolonged venous stasis caused by bed rest, cardiac failure, obesity, and dehydration is another risk factor in critically ill patients in the intensive care unit ${ }^{21)}$. Although recent reports have shown that dehydration is a potential risk factor for worse clinical outcomes in AIS, the relationship between dehydration and the risk of venous thromboembolism in AIS has not been clearly identified ${ }^{20,28)}$. The aim of this study was to investigate the relationship between dehydration, as measured by the blood urea nitrogen (BUN)/creatinine (Cr) ratio, and the risk of VTE in patients with AIS.

\section{MATERIALS AND METHODS}

This retrospective, single-center study included AIS patients who were admitted to our stroke unit between January 1,2012 and December 31, 2013. The study protocol was approved by the Institutional Review Board of the College of Medicine, The Catholic University of Korea (HC14RISI0035).

AIS was diagnosed based on clinical signs, symptoms, and brain magnetic resonance imaging (MRI) at admission. Information collected at initial assessment included the following : demographic characteristics, stroke severity, vascular risk factors, comorbidities, and baseline laboratory results from blood samples collected on the day of admission (blood cell counts, lipid profile, BUN level, Cr level, serum osmolality [SO], international normalized ratio [INR], erythrocyte sedimentation rate [ESR], and C-reactive protein [CRP] level).

The severity of the patients' neurological deficits was evaluated using the National Institutes of Health Stroke Scale (NIHSSS) on the day of admission. Stroke severity was defined as moderate (NIHSS score of 8 to 19) or severe (NIHSS score of 20 or more $)^{9)}$. For each patient, the severity of lower extremity weakness and range of motion were reassessed three days after admission in a standardized examination performed by experienced rehabilitation staff. Manual muscle test (MMT) results were classified as follows : grade 5, movement against gravity plus full resistance; grade 4 , movement against gravity plus some resistance; grade 3 , complete range of motion against gravity with no resistance; grade 2 , full or partial range of motion with no gravity; grade 1 , slight contractility without any movement; and grade 0 , no evidence of contractility (complete paralysis).
The inclusion criteria for this study were as follows : 1) diagnosis of AIS based on clinical presentation and diffusionweighted MRI; 2) AIS diagnosed within 72 hours of symptom onset; and 3) AIS accompanied by lower extremity weakness. The exclusion criteria were the presence of any of the following : 1) transient ischemic stroke; 2) hemorrhagic stroke; 3) age $<18$ or $>88$; 4) current treatment with anticoagulation therapy; 5) active cancer; 6) hospital stay $<2$ weeks; 7) history of DVT or PE; 8) comorbidities influencing neurological status such as seizures or acute respiratory distress; 9) severe stroke (NISSS score $\geq 20$ ); 10) immobility before stroke onset; 11) hormone replacement therapy; and 12) history of heart failure, chronic obstructive pulmonary disease, or renal failure.

Early management of AIS was performed according to guideline recommendations ${ }^{9}$. We administered dual antiplatelet therapy consisting of treatment with aspirin and clopidogrel while the patients were hospitalized. Pharmacologic prophylaxis, such as unfractionated heparin and low molecular weight heparin (LMWH), was not routinely used for VTE prevention. Thromboembolic deterrent stockings were supplied to all patients. Patients diagnosed with VTE were treated with full doses of LMWH. An inferior vena cava filter was also placed if needed.

Patients were divided into two groups : VTE and non-VTE. The VTE group was classified as individuals who were diagnosed with DVT or PE at any time during hospitalization. Diagnosis of VTE was based on clinical and radiological findings. The presence of DVT was confirmed by a lower extremity computed tomography scan, whereas PE was confirmed by computed pulmonary angiography showing the presence of pulmonary emboli or a ventilation-perfusion scan. Diagnostic testing was performed in patients who experienced clinical symptoms suspicious for VTE such as pain, tenderness, swelling, warmth, redness, discoloration, surface vein distention, Homans' sign or Luck's sign in one or both legs, cyanosis, chest pain, or respiratory distress.

\section{Statistical analysis}

Categorical variables were described in terms of numbers and percentages, and continuous variables were described as mean \pm standard deviation when the data were distributed normally. Categorical variables were compared using the Chisquare test or Fisher's exact test, and the medians were compared using Student's t-test or the Wilcoxon rank sum test. 
Logistic regression analysis was used to compute unadjusted and multivariable-adjusted odds ratios (ORs) for dichotomous outcomes of the VTE or non-VTE group. ORs are presented together with their associated $95 \%$ confidence intervals (CIs). The threshold for statistical significance was set at $p<0.05$. All statistical analyses were performed using Statistical Package for the Social Sciences software version 18 (SPSS Inc., Chicago, IL, USA).

\section{RESULTS}

Of the 182 patients included in the study, 165 (90.7\%) were not diagnosed with VTE (non-VTE group) and 17 (9.3\%) were diagnosed with VTE. Among the 17 patients who were diagnosed with VTE, two had accompanying DVT and PE. Table 1 lists the baseline clinical and demographic characteristics of both groups. VTE was diagnosed at a mean of 21 days (range, 11-39 days). The patients in the VTE group were older and a higher proportion was female, but the differences between the groups were not statistically significant. Body mass index, history of smoking, and medical history such as diabetes mellitus, hypertension, hyperlipidemia, atrial fibrillation, and stroke were similar in both groups.

The VTE group presented with higher NIHSS scores at admission compared with patients in the non-VTE group; however, these findings were not statistically significant (NIHSS : 6.5 vs. $8.4, p=0.131$; NIHSS $\geq 8: 36 \%$ vs. $53 \%, p=0.163$ ). Laboratory parameters (blood cell counts, lipid profile, BUN level, Cr level, BUN/Cr ratio, SO, SO>295, INR, ESR, and CRP level) on admission were similar in the two groups. However, the proportion of patients with BUN/Cr ratio $>15$ was significantly different between non-VTE and VTE groups (61\% vs. 94\%, OR 10.1, 95\% CI 1.31-78.31, $p=0.007$; Fig. 1). Additionally, the VTE group showed more severe lower extremity weakness and more frequent range of motion (ROM) limitation compared with patients in the non-VTE group, although these differences were not significant (weakness grade : 2.5 vs. 1.3, $p=0.07$; ROM limitation : $53 \%$ vs. $64 \%, p=0.346$ ). However, when a cutoff of grade $<3$ for lower extremity weakness was used, the non-VTE and VTE groups were significantly different (40\% vs. $76 \%$, OR 5, 95\% CI 1.56-16, $p=0.004$; Table 2).

In a regression analysis including relevant confounders and potential predictors of age, sex, stroke severity, low limb weak- ness, ROM limitation on admission, diabetes, hypertension, hyperlipidemia, atrial fibrillation, smoking, previous stroke,

Table 1. Comparisons of the baseline characteristics of patients in the VTE and non-VTE groups

\begin{tabular}{|c|c|c|c|}
\hline & $\begin{array}{l}\text { Non-VTE } \\
(n=165)\end{array}$ & $\begin{array}{c}\text { VTE } \\
(n=17)\end{array}$ & $p$-value \\
\hline \multicolumn{4}{|l|}{ Baseline variable } \\
\hline Age & $68.6 \pm 11.2$ & $74.1 \pm 10.4$ & 0.054 \\
\hline Female & $72(43)$ & $11(65)$ & 0.097 \\
\hline $\mathrm{BMI}\left(\mathrm{kg} / \mathrm{m}^{2}\right)$ & $23.5 \pm 3.8$ & $22.9 \pm 3.3$ & 0.55 \\
\hline \multicolumn{4}{|l|}{ Medical history } \\
\hline Diabetes mellitus & $56(34)$ & $4(23)$ & 0.589 \\
\hline Hypertension & $111(67)$ & $11(64)$ & 0.83 \\
\hline Hyperlipidemia & $29(18)$ & $4(23)$ & 0.517 \\
\hline Atrial fibrillation & $28(17)$ & $3(18)$ & 0.944 \\
\hline CVA & $39(23)$ & $3(17)$ & 0.766 \\
\hline Smoking & $44(26.7)$ & $4(23.5)$ & 0.78 \\
\hline t-PA & $25(15.2)$ & $5(29)$ & 0.164 \\
\hline \multicolumn{4}{|l|}{ Laboratory values } \\
\hline Hemoglobin (g/dL) & $13.6 \pm 2$ & $13.5 \pm 1.3$ & 0.92 \\
\hline $\begin{array}{l}\text { White blood cell count } \\
\qquad\left(\times 10^{6} \text { per } \mathrm{mL}\right)\end{array}$ & $9.33 \pm .69$ & $8.28 \pm 2.65$ & 0.254 \\
\hline $\begin{array}{l}\text { Platelet count } \\
\qquad\left(\times 10^{3} \text { per } \mathrm{mL}\right)\end{array}$ & $228.2 \pm 82.07$ & $217.59 \pm 52.51$ & 0.603 \\
\hline Triglyceride (mg/dL) & $149.9 \pm 97.9$ & $139.3 \pm 93.6$ & 0.67 \\
\hline Total cholesterol (mg/dL) & $186.2 \pm 46.3$ & $174.3 \pm 42.7$ & 0.31 \\
\hline $\mathrm{HDL}(\mathrm{mg} / \mathrm{dL})$ & $47.1 \pm 12.9$ & $47.5 \pm 15.4$ & 0.88 \\
\hline $\mathrm{LDL}(\mathrm{mg} / \mathrm{dL})$ & $117.8 \pm 47.6$ & $106.3 \pm 34.5$ & 0.333 \\
\hline $\mathrm{ESR}(\mathrm{mm} / \mathrm{hr})$ & $18.3 \pm 13.8$ & $14.4 \pm 10.7$ & 0.264 \\
\hline Bun & $16 \pm 10.3$ & $15.5 \pm 4.2$ & 0.854 \\
\hline $\mathrm{Cr}$ & $0.96 \pm 0.86$ & $0.75 \pm 0.18$ & 0.316 \\
\hline Bun/Cr ratio & $18.53 \pm 7.64$ & $21.62 \pm 7.83$ & 0.115 \\
\hline Serum osmol & $295.3 \pm 9.4$ & $297.4 \pm 8.1$ & 0.407 \\
\hline $\begin{array}{l}\text { C-reactive protein } \\
(\mathrm{mg} / \mathrm{dL})\end{array}$ & $12.9 \pm 34.2$ & $5.1 \pm 13$ & 0.355 \\
\hline INR & $1.62 \pm 7.55$ & $1.16 \pm 0.51$ & 0.802 \\
\hline NIHSS & $6.5 \pm 5.1$ & $8.4 \pm 5.5$ & 0.131 \\
\hline Lower limb weakness & $2.5 \pm 1.3$ & $1.6 \pm 0.9$ & 0.07 \\
\hline
\end{tabular}

Values are presented as mean \pm standard deviation or number (\%). $p$ value represents comparisons between the non-deep vein thrombosis and deep vein thrombosis groups using Fisher's exact test and the Wilcoxon rank sum test. VTE : venous thromboembolism, BMI : body mass index, CVA : cerebrovascular accident, $\mathrm{HDL}$ : high-density lipoprotein, LDL : low-density lipoprotein, BUN : blood urea nitrogen, $\mathrm{Cr}$ : creatinine, INR : international normalized ratio, NIHSS : National Institutes of Health Stroke Scale 
Dehydration and Deep Vein Thrombosis after Acute Cerebral Infarction I Kim H, et al.

Table 2. Analysis of risk factors for VTE is patients with acute ischemic stroke by simple logistic regression

\begin{tabular}{lccccc}
\hline & Non-VTE $(\mathbf{n = 1 6 5 )}$ & VTE $(\mathbf{n = 1 7 )}$ & p-value & OR* $^{*}$ & $\mathbf{9 5 \%}$ Cl \\
\hline Bun $/$ Cr ratio $>15$ & $121(61)$ & $16(94)$ & 0.007 & 10.1 & $1.31-78.31$ \\
Serum osmol $>295$ & $84(51)$ & $12(70)$ & 0.122 & 2.31 & $0.78-6.86$ \\
NIHSS $\geq 8$ & $59(36)$ & $9(53)$ & 0.163 & 2.02 & $0.74-5.51$ \\
Lower limb weakness $<3$ & $65(40)$ & $13(76)$ & 0.004 & 5 & $1.56-16$ \\
ROM limitation & $87(53)$ & $11(64)$ & 0.346 & 1.64 & $0.58-4.65$ \\
\hline
\end{tabular}

Values are presented as number (\%) unless otherwise indicated. *The OR represents comparisons between the non-VTE and VTE groups using simple logistic regression. VTE : venous thromboembolism, OR : odds ratio, $\mathrm{Cl}$ : confidence interval, BUN : blood urea nitrogen, $\mathrm{Cr}$ : creatinine, NIHSS : National Institutes of Health Stroke Scale, ROM : range of motion

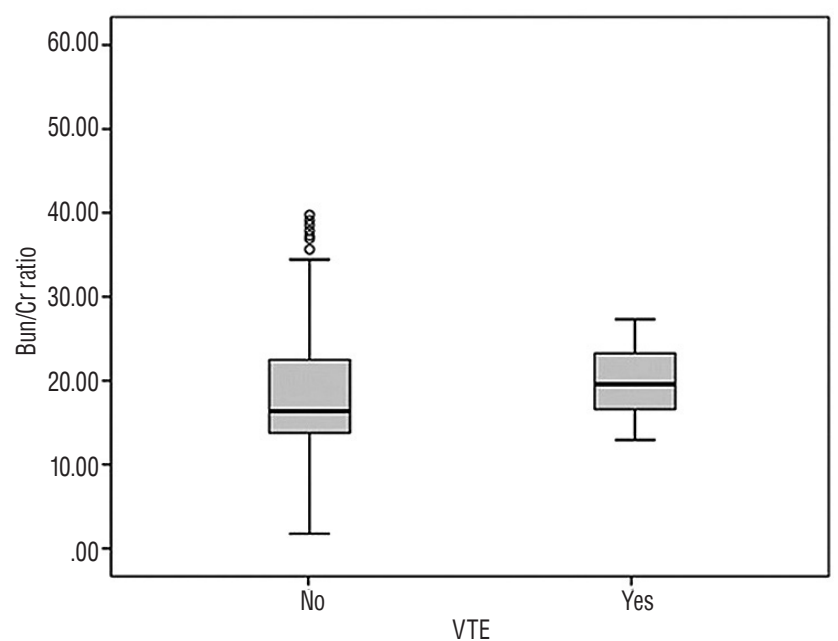

Fig. 1. Representative box plot showing the distribution of $\mathrm{BUN} / \mathrm{Cr}$ ratio between non-VTE and VTE groups. BUN : blood urea nitrogen, $\mathrm{Cr}$ : creatinine, VTE : venous thromboembolism.

and laboratory findings. Two parameters were identified as predictive factors for VTE : BUN/Cr ratio $>15$ on admission (OR 8.75, 95\% CI 1.11-68.49, $p=0.039$ ) and the presence of lower extremity weakness of grade $<3$ (OR $4.38,95 \%$ CI 1.3414.26, $p=0.014$; Table 3).

\section{DISCUSSION}

In this study, VTE was reported in 9.3\% of AIS patients with prolonged hospitalization. We compared the demographic and clinical characteristics of 17 patients with VTE and 165 patients without VTE. Multivariate analyses showed that lower limb weakness (poor, trace, or zero using the MMT grading system) and dehydration (defined by BUN/Cr ratio
Table 3. Multivariate regression analysis for predictors of venous thromboembolism

\begin{tabular}{lccc}
\hline & Adjusted OR* & 95\% Cl & p-value $^{\dagger}$ \\
\hline Bun $/$ Cr ratio $>15$ & 8.75 & $1.12-68.49$ & 0.039 \\
\hline Lower limb weakness $<3$ & 4.38 & $1.34-14.26$ & 0.014 \\
\hline
\end{tabular}

*The OR was adjusted using age and sex. ${ }^{\dagger}$ The $p$ value represents comparisons between the non-VTE and VTE groups using multiple logistic regression. OR : odds ratio, $\mathrm{Cl}$ : confidence interval, BUN : blood urea nitrogen, $\mathrm{Cr}$ : creatinine, VTE : venous thromboembolism

>15) were significant independent risk factors for VTE.

The incidence of VTE in immobilized post-stroke patients ranges from $10 \%$ to $75 \%{ }^{4}$. Such a wide variation may be attributed to differences in diagnostic methods and timing, the inclusion of symptomatic or asymptomatic patients or of immobilized or mobile patients, and stroke severity among different studies ${ }^{13,16,24)}$. According to several previous studies, Asians appear to be less prone to development of VTE than other races ${ }^{36)}$. We found that VTE developed in $9.3 \%$ of our patients. In a recent large trial (effectiveness of thigh-length graduated compression stockings to reduce the risk of deep vein thrombosis after stroke [CLOTS trial 1]), symptomatic or asymptomatic VTE was found in $10.0 \%$ of the patients allocated to use thigh-length graduated compression stockings and in $10.5 \%$ of the patients allocated to not use graduated compression stockings; these rates are in agreement with our results ${ }^{10)}$.

Our finding that the risk of VTE is increased in patients with severe lower limb weakness is supported by multiple previous studies. In general, patients who have suffered an ischemic stroke are at increased risk for VTE. Additionally, patients with lower extremity paresis face greater risks of developing VTE because limb paresis and immobilization re- 
duce muscle-pumping activity in the legs, thereby resulting in reduced venous blood flow. Thus, partial or total immobilization due to lower limb paresis in stroke patients is considered to be the greatest risk factor for $\mathrm{VTE}^{13)}$. A recent meta-analysis of epidemiological studies found that the risk of VTE in immobilized stroke patients is double that in non-immobilized stroke patients ${ }^{25}$. Risk factors of VTE in the general population are generally recognized to include older age, obesity, cigarette smoking, hormonal replacement, pregnancy, previous medical illness (such as cancer, COPD, congestive heart failure, thrombophilia, smoking, and hypertension), stroke with limb paresis, and recent surgery. In our study, all AIS patients had limb paresis, and approximately $40 \%$ of the patients were unable to resist gravity, confirming the higher risk of VTE among patients with more severe lower limb weakness.

Dehydration is a frequent clinical problem and a marker of poor prognosis in many clinical conditions, including pneumonia and myocardial infarction. Dehydration is also a marker of clinical deterioration in AIS and may be caused by decreased oral water intake due to disturbed consciousness or dysphagia. Although several clinical and laboratory tests have been applied for accurate diagnosis of dehydration severity, there is no definite absolute cutoff value. Weight loss, skin turgor, deep breathing, and capillary filling time have some limitations because baseline levels were not evaluated ${ }^{31,34)}$. Urea concentration $\geq 100 \mathrm{mg} / \mathrm{dL}$, bicarbonate level $\geq 15 \mathrm{mEq} / \mathrm{L}$, and serum osmolality have also been used for the diagnosis of dehydration severity ${ }^{11,37}$. One observational study found an association between elevated osmolality $(>296 \mathrm{mOsm} / \mathrm{kg}$ ) on admission following acute stroke ( $90 \%$ ischemic) and mortality within three months after adjusting for potential confounding factors ${ }^{6}$. Additionally, dehydration is assumed to predispose patients to VTE. Although there is little evidence indicating dehydration as an independent risk factor for VTE in AIS patients ${ }^{3}$, dehydration has been suggested to increase the risk of VTE during long-duration and long-distance air travel, a phenomenon known as economy class syndrome ${ }^{2)}$. Dehydration reduces total plasma volume, decreases cardiac output, reduces blood flow to muscle, and can change blood rheology (an increase in local hematocrit and blood viscosity $)^{12}$. Co-existence of two of three major risk factors of Virchow's triad (alterations in blood flow, vascular endothelial injury, and alterations in the constitution of the blood) predisposes some dehydrated patients to a heightened risk of $\mathrm{VTE}^{35)}$.
In our study, dehydration measured using a biochemical index $(\mathrm{BUN} / \mathrm{Cr}$ ratio $>15)$ less than three days post-stroke was significantly associated with the development of VTE in univariate and multivariable analyses, with an OR of 8.75 by multivariable analysis, indicating that dehydration is an independent risk factor for the development of VTE in AIS patients. $\mathrm{BUN} / \mathrm{Cr}$ ratio is one of the common laboratory tests used to evaluate prerenal azotemia, acute tubular necrosis, and dehydration, with a threshold of $20^{1,5,19,26)}$. Recently, several studies have shown that $\mathrm{BUN} / \mathrm{Cr}$ ratio $>15$ is a reference marker for early neurological deterioration, three-month outcome, and hospital cost in AIS patients ${ }^{720,29)}$. Our study provides added significance of BUN/Cr ratio $>15$ regarding VTE in AIS, although the mechanisms by which initial hydration status and hydration therapy can influence the incidence of VTE, mortality, or functional recovery in ischemic stroke remain unclear ${ }^{15,23)}$. Fluid replacement may reduce the occurrence of dehydration and increase cerebral blood flow and oxygen delivery, and maintaining systemic blood pressure after acute stroke may contribute to improved clinical outcomes after $\mathrm{AIS}^{27,32}$. Also, given the association between VTE and dehydration after AIS, our findings suggest that encouraging fluid replacement may also reduce the likelihood of developing VTE especially in dehydrated patients with AIS presenting with BUN/Cr ratio $>15$. No evidence-based method for predicting the occurrence of VTE after stroke currently exists, and our findings suggest that a high Bun/Cr ratio (BUN/Cr $>15$ ) in the acute stage is an independent predictor of VTE in AIS patients. Although there is no single 'gold standard' clinical or laboratory measure of the hydration status, BUN and Cr levels are routinely measured in all medical fields. Our study thus provides a new perspective on predicting the occurrence of VTE in AIS patients, and this cutoff may apply to all stroke patients. Our findings support the use of prophylactic anticoagulation if the Bun/Cr ratio is elevated regardless of the AIS mechanism and stroke severity.

Although our findings are preliminary, based on our results, physicians may consider maintaining hydration in previously dehydrated patients with severe lower limb weakness in the early stage of ischemic stroke in order to reduce the chance of VTE. Treatment with LMWH is the preferred method for preventing VTE regardless of the subtype of ischemic stroke. A larger-scale randomized controlled trial is required to replicate the findings of this preliminary study. 
There are several limitations of this study : 1) we cannot draw strong conclusions because the sample size was small and the control group was retrospectively selected; 2) there was a lack of uniformity in the thromboprophylaxis administered to the patients; 3) we excluded patients with severe stroke (NIHSS $\geq 20$ ) or a short hospital stay, regardless of the reason; 4) we did not perform routine diagnostic procedures such as ultrasonography to identify cases of VTE and thus might have missed clinically silent cases; 5) we were not able to perform autopsies on patients who died during the study period; and 6) only serum markers were used to measure dehydration.

\section{CONCLUSION}

In conclusion, our study shows that severe lower limb paresis is associated with VTE. Moreover, an elevated serum BUN/ $\mathrm{Cr}$ ratio $(>15)$ is independently associated with an increased risk of VTE. Therefore, physicians should be aware of the danger of dehydration and be attentive to patients with higher BUN/Cr ratios and severe leg paresis. Further investigations of the pathophysiology of VTE are needed, and a larger controlled study is required to assess whether these results hold true in a larger patient population.

\section{References}

1. Agrawal M, Swartz R : Acute renal failure. Am Fam Physician 61 : 2077-2088, 2000

2. Anderson FA Jr, Spencer FA : Risk factors for venous thromboembolism. Circulation 107(23 Suppl 1) : 19-116, 2003

3. Ansell JE : Air travel and venous thromboembolism--is the evidence in? N Engl J Med 345 : 828-829, 2001

4. Bembenek J, Karlinski M, Kobayashi A, Czlonkowska A : Early strokerelated deep venous thrombosis: risk factors and influence on outcome. J Thromb Thrombolysis 32 : 96-102, 2011

5. Bennett JA, Thomas $V$, Riegel $B$ : Unrecognized chronic dehydration in older adults: examining prevalence rate and risk factors. J Gerontol Nurs 30 : 22-28; quiz 52-53, 2004

6. Bhalla A, Sankaralingam $S$, Dundas $R$, Swaminathan $R$, Wolfe $C D$, Rudd $A G$ : Influence of raised plasma osmolality on clinical outcome after acute stroke. Stroke 31 : 2043-2048, 2000

7. Bhatia K, Mohanty S, Tripathi BK, Gupta B, Mittal MK : Predictors of early neurological deterioration in patients with acute ischaemic stroke with special reference to blood urea nitrogen (BUN)/creatinine ratio \& urine specific gravity. Indian J Med Res 141 : 299-307, 2015

8. Bounds JV, Wiebers DO, Whisnant JP, Okazaki H : Mechanisms and timing of deaths from cerebral infarction. Stroke $12: 474-477,1981$

9. Broderick JP, Palesch YY, Demchuk AM, Yeatts SD, Khatri P, Hill MD, et al. : Endovascular therapy after intravenous t-PA versus t-PA alone for stroke. N Engl J Med 368 : 893-903, 2013

10. CLOTS Trials Collaboration, Dennis M, Sandercock PA, Reid J, Graham C, Murray $G$, et al. : Effectiveness of thigh-length graduated compression stockings to reduce the risk of deep vein thrombosis after stroke (CLOTS trial 1): a multicentre, randomised controlled trial. Lancet 373 : 19581965, 2009

11. Goldman RD, Friedman JN, Parkin PC : Validation of the clinical dehydration scale for children with acute gastroenteritis. Pediatrics 122 : 545 549, 2008

12. González-Alonso J, Calbet JA, Nielsen B : Muscle blood flow is reduced with dehydration during prolonged exercise in humans. J Physiol 513(Pt 3) : 895-905, 1998

13. Harvey RL : Prevention of venous thromboembolism after stroke. Top Stroke Rehabil $10: 61-69,2003$

14. Hull RD : Revisiting the past strengthens the present: an evidence-based medicine approach for the diagnosis of deep venous thrombosis. Ann Intern Med 142 : 583-585, 2005

15. Jauch EC, Saver JL, Adams HP Jr, Bruno A, Connors JJ, Demaerschalk BM, et al. : Guidelines for the early management of patients with acute ischemic stroke: a guideline for healthcare professionals from the American Heart Association/American Stroke Association. Stroke 44 : 870 947, 2013

16. Kelly J, Rudd A, Lewis RR, Coshall C, Moody A, Hunt BJ : Venous thromboembolism after acute ischemic stroke: a prospective study using magnetic resonance direct thrombus imaging. Stroke 35 : 2320-2325, 2004

17. Kelly J, Rudd A, Lewis RR, Coshall C, Parmar K, Moody A, et al. : Screening for proximal deep vein thrombosis after acute ischemic stroke: a prospective study using clinical factors and plasma D-dimers. J Thromb Haemost 2 : 1321-1326, 2004

18. Landi G, D'Angelo A, Boccardi E, Candelise L, Mannucci PM, Morabito A, et al. : Venous thromboembolism in acute stroke. Prognostic importance of hypercoagulability. Arch Neurol 49 : 279-283, 1992

19. Leibovitz A, Baumoehl Y, Lubart E, Yaina A, Platinovitz N, Segal R Dehydration among long-term care elderly patients with oropharyngeal dysphagia. Gerontology 53 : 179-183, 2007

20. Liu CH, Lin SC, Lin JR, Yang JT, Chang YJ, Chang CH, et al. : Dehydration is an independent predictor of discharge outcome and admission cost in acute ischaemic stroke. Eur J Neurol 21 : 1184-1191, 2014

21. Marik PE, Andrews L, Maini $B$ : The incidence of deep venous thrombosis in ICU patients. Chest $111: 661-664,1997$

22. Noel $P$, Gregoire $F$, Capon A, Lehert $P$ : Atrial fibrillation as a risk factor for deep venous thrombosis and pulmonary emboli in stroke patients. Stroke 22 : 760-762, 1991

23. O'Neill PA, Davies I, Fullerton KJ, Bennett D : Fluid balance in elderly patients following acute stroke. Age Ageing 21 : 280-285, 1992 
24. Oczkowski WJ, Ginsberg JS, Shin A, Panju A : Venous thromboembolism in patients undergoing rehabilitation for stroke. Arch Phys Med Rehabil $73:$ 712-716, 1992

25. Pottier $P$, Hardouin JB, Lejeune $S$, Jolliet $P$, Gillet B, Planchon B : Immobilization and the risk of venous thromboembolism. A meta-analysis on epidemiological studies. Thromb Res 124 : 468-476, 2009

26. Riccardi A, Chiarbonello B, Minuto P, Guiddo G, Corti L, Lerza R : Identification of the hydration state in emergency patients: correlation between caval index and BUN/creatinine ratio. Eur Rev Med Pharmacol Sci 17 : 1800-1803, 2013

27. Rønning OM, Guldvog B : Stroke unit versus general medical wards, II: neurological deficits and activities of daily living: a quasi-randomized controlled trial. Stroke 29 : 586-590, 1998

28. Rowat A, Graham C, Dennis M : Dehydration in hospital-admitted stroke patients: detection, frequency, and association. Stroke 43 : 857-859, 2012

29. Schrock JW, Glasenapp M, Drogell K : Elevated blood urea nitrogen/creatinine ratio is associated with poor outcome in patients with ischemic stroke. Clin Neurol Neurosurg 114 : 881-884, 2012

30. Skaf E, Stein PD, Beemath A, Sanchez J, Bustamante MA, Olson RE : Venous thromboembolism in patients with ischemic and hemorrhagic stroke. Am J Cardiol 96 : 1731-1733, 2005
31. Steiner MJ, DeWalt DA, Byerley JS : Is this child dehydrated? JAMA $291: 2746-2754,2004$

32. Sulter G, De Keyser J : From stroke unit care to stroke care unit. J Neurol Sci $162: 1-5,1999$

33. Turpie AG, Levine $M N$, Hirsh J, Carter CJ, Jay RM, Powers PJ, et al. : Double-blind randomised trial of Org 10172 low-molecular-weight heparinoid in prevention of deep-vein thrombosis in thrombotic stroke. Lancet 1 : 523-526, 1987

34. Vega RM, Avner JR : A prospective study of the usefulness of clinical and laboratory parameters for predicting percentage of dehydration in children. Pediatr Emerg Care 13 : 179-182, 1997

35. Virchow R : Thrombose und embolie. Gefässentzündung und septische infektion in Virchow R (ed) : Gesammelte abhandlungen zur wissenschaftlichen medicin. Frankfurt : Verlag von Meidinger Sohn \& Comp, 1856, pp219-732

36. White RH, Zhou H, Romano PS : Incidence of idiopathic deep venous thrombosis and secondary thromboembolism among ethnic groups in California. Ann Intern Med 128 : 737-740, 1998

37. Yilmaz K, Karaböcüoglu M, Citak A, Uzel N : Evaluation of laboratory tests in dehydrated children with acute gastroenteritis. J Paediatr Child Health 38 : 226-228, 2002 